\title{
Effects of Age and Sex on Sickle Cell Disease Avascular Necrosis
}

\author{
Al-Jafar $\mathrm{H}^{* 1}$, AlFadhli $\mathrm{S}^{2}$, Al-Feeli $\mathrm{M}^{3}$, Ali $\mathrm{A}^{4}$ and Alhajri F \\ ${ }^{1}$ Department of Haematology, Amiri Hospital, Kuwait \\ ${ }^{2}$ Medical laboratory Sciences, Faculty of Allied Health Sciences, Kuwait University \\ ${ }^{3}$ Department of Nuclear medicine, Mubarak Al-Kabeer Hospital, Jabria, Kuwait \\ ${ }^{4}$ Department of Orthopedic Surgeon, Al-Razi Hospital, Shuwaikh, Kuwait \\ ${ }^{5}$ Department of Clinical Radiology, Amiri Hospital, Gulf street, Kuwait City, Kuwait
}

${ }^{*}$ Corresponding author: Al-Jafar H, Consultant Haematologist, Department of Haematology, Amiri Hospital, Kuwait, E-mail: cbc9@hotmail.com

\author{
Citation: Al-Jafar H, AlFadhli S, Al-Feeli M, Ali A, Alhajri F (2016) Effects of Age and Sex on Sickle Cell \\ Disease Avascular Necrosis. J Hematol Blood Disord 2(1): 104. doi: 10.15744/2455-7641.2.104
}

Received Date: March 01, 2016 Accepted Date: April 14, 2016 Published Date: April 15, 2016

\begin{abstract}
Introduction: Sickle cell disease (SCD) is a hemoglobinopathy. Based on genotypes, it is classified into sickle cell thalassemia (SCTh) and sickle cell anemia (SCA). Red blood cells in SCD are less pliable; therefore, these cells distort to a sickle shape that leads to tissue infarctions and progressive bone avascular necrosis (AVN).

Aim: To study the significant effects of age and sex on AVN in SCD.

Methods: Data of 68 patients with SCD were collected (25 and 43 patients with SCTh and SCA, respectively). Their age ranged from 4 to 78 years. Statistical test conducted to analyze the significant effects of age and sex on AVN in all the 68 patients.

Results: AVN prevalent is $76 \%$ and $72 \%$ in patients with SCTh and SCA, respectively. In SCTh age effect on AVN in SCTh ( $p=0.546$ ) and Sex effect on AVN ( $p=0.013$ ), while in SCA age effect on AVN ( $p=0.783$ ) and Sex effect on AVN in SCA ( $p=0.334)$. The HPLC results showed HbF mean values in SCTh 12\%, while in SCA 17\%. HPLC results showed HbA2 mean values in SCTh $4.9 \%$ (4.9 in children, 4.9 in adults, 4.8 in females and 4.9 in males), and in SCA 2.1\% (1.9 in children, 2.4 in adults, 2.1 in females and 2.3 in males.

Conclusion: In age group, no significant relation found between age and AVN in both SCTh and SCA. In sex group no significant relationship found between sex and SCA. However, a significant result detected between AVN and sex in SCTh; males have more tendency to develop AVN than females in both SCTh and SCA. HbF was higher in SCA than in SCTh due to hydroxyurea treatment and AVN prevalence was also less in SCA.
\end{abstract}

Keywords: Sickle cell anemia; Sickle cell thalassemia; Bone scintigraphy; Osteonecrosis; Sex

\section{Introduction}

Sickle cell disease (SCD) is a heterogeneous disease, which affects men and women equally [1]. Patients with SCD experience multiple disease-related complications [2]. Although anemia is a common sign in SCD because of the short lifespan of RBCs, the primary symptomatic manifestation is a pain crisis with wide variation in intensity, quality, duration, and persistence. Essentially, SCD crises occur when partially or totally deoxygenated hemoglobin $(\mathrm{Hb})$ molecules distort their normal disk shape and lead to rigid, sticky, sickle-shaped cells that obstruct small blood vessels and cause vaso-occlusive crises (VOC ), which further leads to disruption of oxygen supply to body tissues [3]. The recurrent acute VOC leads to chronic pain and organ damage in the end. It potentially affects all the organ systems, particularly the bones. The complete disruption of vascular supply to the articular surfaces and ends of long bones, particularly the femoral and humeral head and spine, results in avascular necrosis (AVN) [4]. It is a devastating complication of SCD and is highly prevalent in adults with SCD [5].

Several studies have examined risk factors for AVN development in SCD including recurrent VOC, high $\mathrm{Hb}$ levels, low $\mathrm{Hb} \mathrm{F}$ levels, vitamin D deficiency, and alpha thalassemia trait [6,7]. However, very few studies have examined the relationship of this complication with sex and age. Significant sex differences in morbidity and mortality have been reported in adults with SCD [8]. Garvin observed that morbidity apparently increased in men; further, sex-related differences in some acute and chronic complications of this disorder were suggested [9]. In a cooperative study on SCD, Platt et al. reported median death ages for men and women as 42 and 48 years, respectively; however, this difference was greater than that observed in black control subjects [10]. In 2011, Adekile et al. observed that femoral head AVN is the most common complication in Kuwaiti patients with SCD [11], and he reported that it was more common in men than in women in SCD. We studied the significant effects of age and sex on AVN development in patients with SCD in Kuwait. 


\section{Materials and Methods}

This is a retrospective study in which the data were collected from our institute's electronic database in the years 2008-2015 for 68 patients with SCD. Both male and female patients with age from 4 to 78 years were included; of these, 41 underwent bone scintigraphy (BSc), and 27 underwent magnetic resonance imaging (MRI) for different reasons. Our exclusion criteria were bone diseases due to factors other than SCD such as tumors and endocrinology disorders, steroid therapy, irradiation treatment, or alcohol consumption. The patients were divided into two groups based on the SCD genotype as follows: the Hb S-thalassemia (SCTh) and HbSS (SCA) groups. In both groups, AVN was tested for significant relationships with age (child and adult) and sex (female and male). The patients' medical charts were reviewed for complete blood count, high performance liquid chromatography (HPLC), and AVN. Bone scintigraphs were retrieved electronically for all patients in this study and were examined for AVN. Infinia Hawkeye 4.0 SPECT-CT camera (GE Healthcare) was used. For bone scans, technetium-99m hydroxymethylene diphosphonate (HDP) was administered intravenously. For MRI, Echelon apparatus was used. Statistical analysis was performed by using SPSS; the crosstab statistical analysis test was conducted to find the significant values of age and sex in both SCA and SCTh groups.

\section{Results}

Bone AVN is a frequent outcome in patients with SCD. The objective of the current study was to check if age and sex were associated risk factors for AVN development in patients with SCD including both the genotypes SCA and SCTh; the patients underwent BSc and MRI for different reasons. We collected the data of 68 subjects that represented both the SCD genotypes SCTh and SCA. In all the patients, AVN was a common complication observed in both the types of SCD.

In SCTh 93.3\% male patients compared to 50\% female patients developed AVN. Similarly, among patients with SCA, 78.3\% male patients compared to 65\% female patients developed AVN. Number of AVN observed in 25 patients with SCTh 19 (76\%). Number of AVN observed in 43 patients with SCA 31 (72\%). However in SCTh the age effect on AVN in SCTh ( $\mathrm{p}=0.546)$ and the Sex effect on AVN ( $\mathrm{p}=0.013)$. In SCA the age effect on AVN in SCA ( $\mathrm{p}=0.783)$ and the Sex effect on AVN in SCA ( $\mathrm{p}=0.334)$ (Table 1).

\begin{tabular}{|c|c|c|c|c|c|c|c|c|}
\hline & \multicolumn{2}{|c|}{$\begin{array}{c}\text { Effect of age on AVN } \\
\text { in SCTh }\end{array}$} & \multicolumn{2}{|c|}{$\begin{array}{c}\text { Effect of gender on AVN } \\
\text { in SCTh }\end{array}$} & \multicolumn{2}{|c|}{$\begin{array}{c}\text { Effect of age on AVN } \\
\text { in SCA }\end{array}$} & \multicolumn{2}{|c|}{$\begin{array}{c}\text { Effect of gender on } \\
\text { AVN in SCA }\end{array}$} \\
\hline & \multicolumn{4}{|c|}{$\operatorname{SCTh}(N=25)$} & \multicolumn{4}{|c|}{$\operatorname{SCA}(N=43)$} \\
\hline & Child & Adult & Female & Male & Child & Adult & Female & Male \\
\hline AVN & $9(81.8 \%)$ & $10(71.4 \%)$ & $5(50.0 \%)$ & $14(93.3 \%)$ & $9(69.2 \%)$ & $22(73.3 \%)$ & $13(65.0 \%)$ & $18(78.3 \%)$ \\
\hline No AVN & $2(18.2 \%)$ & $4(28.6 \%)$ & $5(50.0 \%)$ & $1(6.7 \%)$ & $4(30.8 \%)$ & $8(26.7 \%)$ & $7(35.0 \%)$ & $5(21.7 \%)$ \\
\hline Total & 11 & 14 & 10 & 15 & 13 & 30 & 20 & 23 \\
\hline P Value & \multicolumn{2}{|c|}{0.546} & \multicolumn{2}{|c|}{0.013} & \multicolumn{2}{|c|}{0.783} & \multicolumn{2}{|c|}{0.334} \\
\hline
\end{tabular}

Table 1: Effects of age and sex as possible associated risk factors for AVN development in patients

The HPLC results (Table 2) showed the HbF mean values in SCTh 12\% (13.6 in children, 10.6 in adults, 12.1 in females and 11.9 in males), while it is in SCA $17 \%$ (20.5 in children, 15.2 in adults, 18.2 in females and 15.8 in males). Further the HPLC results showed the HbA2 mean values in SCTh $4.9 \%$ (4.9 in children, 4.9 in adults, 4.8 in females and 4.9 in males), while it is in SCA $2.1 \%$ (1.9 in children, 2.4 in adults, 2.1 in females and 2.3 in males.

\begin{tabular}{|c|c|c|c|c|c|c|c|c|}
\hline & Child & Adult & Female & Male & Child & Adult & Female & Male \\
\hline AVN & \multicolumn{4}{|c|}{$\begin{array}{l}\text { SC-Thalassemia Group } \\
(n=9)(n=10)(n=5)(n=14)\end{array}$} & \multicolumn{4}{|c|}{$\begin{array}{c}\text { SC-Anemia Group } \\
(n=9)(n=22)(n=13)(n=18)\end{array}$} \\
\hline $\begin{array}{c}\text { Hb A } \\
\text { mean } \pm S D\end{array}$ & $20.9 \pm 12.6$ & $17.7 \pm 0.00$ & $18.8 \pm 13.7$ & $22.3 \pm 5.69$ & & $0.45 \pm 0.00$ & $0.45 \pm 0.00$ & \\
\hline $\begin{array}{c}\text { Hb F } \\
\text { mean } \pm \text { SD }\end{array}$ & $13.6 \pm 8.20$ & $10.6 \pm 5.40$ & $12.15 \pm 4.38$ & $11.9 \pm 7.76$ & $20.5 \pm 5.56$ & $15.2 \pm 6.99$ & $18.2 \pm 4.69$ & $15.8 \pm 8.23$ \\
\hline $\begin{array}{c}\text { Hb S } \\
\text { mean } \pm \text { SD }\end{array}$ & $72.3 \pm 12.5$ & $82.9 \pm 6.44$ & $71.8 \pm 11.8$ & $80.0 \pm 10.1$ & $77.7 \pm 5.37$ & $80.6 \pm 10.4$ & $79.7 \pm 4.44$ & $79.7 \pm 11.6$ \\
\hline $\begin{array}{c}\mathrm{Hb} \mathrm{A}_{2} \\
\text { mean } \pm \mathrm{SD}\end{array}$ & $4.9 \pm 0.65$ & $4.9 \pm 0.37$ & $4.8 \pm 0.40$ & $4.9 \pm 0.56$ & $1.9 \pm 0.58$ & $2.4 \pm 0.66$ & $2.1 \pm 0.85$ & $2.3 \pm 0.50$ \\
\hline
\end{tabular}

Normal HPLC reference value: $\mathrm{HbA}(96$ - 98), $\mathrm{HbA} 2(1.5-3.5), \mathrm{HbF}<1, \mathrm{HbS}=0$

Table 2: The HPLC result for each group of sickle cell disease patients having avascular necrosis

\section{Discussion}

SCD is a group of disorders that affects both men and women. Those face a chronic disease, with onset in childhood leading to devastating consequences [1]. In spite of the impressive improvements in the survival of patients with SCD, scientific advances in chronic bone diseases are still lagging $[12,13]$. AVN, which refers to bone destruction due to subchondral oxygen deficiency, remains 
the leading cause of crippling disability and the most common manifestation in patients with SCD [14,15]. Approximately 50\% of the individuals with SCD are likely to develop some form of bone fragility syndrome by the age of 35 years [3]. In Jamaica, Lee et al. reported that the likelihood of developing AVN was $82 \%$ between the age of 10 and 29 years [16]. Little attempt has been made to survey age and sex-based differences in AVN in individuals with SCD.

We studied the significant effects of age and sex on AVN in patients with SCD. Pertaining to age, we have observed no significant differences between children and adults in both the sexes affected with SCD. Our finding is not in agreement with the recent studies on SCD that have suggested a lower prevalence (26\%) of AVN among children with Hb SS (mean age, 9.8 years) compared to adults (48.6\%) with a mean age of 26.7 years. In a study by Ware et al. $41 \%$ of adults with Hb SS over the age of 15 years had bone AVN [17]. However, Koduri et al. reported that silent AVN occurred in 41\% children aged 4-28 years with the spine and shoulder being involved in $27 \%$ and $28 \%$ cases, respectively [18]. This indicates that there is an underestimation of AVN prevalence in children because of asymptomatic AVN or insensitive AVN investigation by using radiography [19]. Earlier studies on AVN prevalence in patients with SCD were grossly underestimated, particularly, since $47 \%$ and $79 \%$ patients with hip and shoulder disease, respectively had no symptoms at the diagnosis time [20] for that reason bone scintigraphy is recommended to detect the silent and the multifocal AVN [21]. We screened for AVN in our study by both the following methods: bone nuclear scintigraphy by utilizing the radionuclide technetium- $99 \mathrm{~m}$, which is the most sensitive technique, and MRI, which is considered as the gold standard technique that is the most sensitive and specific one for the diagnosis of this condition. Therefore, our estimation of AVN prevalence in both the adults and children was accurate. Pertaining to sex-based differences in AVN, prevalence in male versus female patients, we observed that the phenotypic heterogeneity of SCA, especially, in terms of AVN development was not linked to sex. The high $\mathrm{Hb}$ F level considered a protective factor in SCD [22] .

The HPLC results showed the mean HbF value in sex groups is higher than normal in both SCTh $12 \%$ (male $11.9 \pm 7.76$, female $12.1 \pm 4.38$ ) and SCA $17 \%$ (male $15.8 \pm 8.23$, female $18.2 \pm 4.69$ ). It is known that Hb F is high in SCTh as part of this genetic disorder. However, the high Hb F in SCA is most likely due to hydroxyurea treatment such patients commenced on. The higher $\mathrm{HbF}$ in SCA correlated with lower AVN 72\% than in SCTh where the AVN was 76\%. Although the HbF was almost similar between both sexes in SCTh and SCA group, but the male was more affected due to, sex factors which going with our hypothesis in this study (Table 2). The HbA2 mean value as expected is within normal in SCA 2.1\%, while it was higher than normal in SCTh $4.9 \%$. This need more research work to detect if it has any influnce on the higher incidence of AVN in SCTh.

Very few studies have shown the high incidence of AVN in male patients, and to our knowledge, none have stressed on sex as a factor associated with AVN and management of patients with SCD. The results of our study indicated a significant relationship between AVN and sex $(p=0.013)$ in SCTh, such that, male patients had more tendency to develop AVN than the female patients did. In patients with SCTh, $93.3 \%$ male patients compared to $50 \%$ female patients developed AVN. Similarly, in patients with SCA, $78.3 \%$ male patients compared to $65 \%$ female patients developed AVN. The female patients with SCTh had a lower tendency to develop AVN (50\%) than the female patients with SCA did (65\%).

To date, there is no well-established consensus among providers on the management of SCD complications partly because of lack of evidence and because of differences in the experience of the providers [23]. We strongly emphasize on the idea of screening all patients with SCD for AVN to monitor these complications; we hope to establish it as a routine protocol for all patients with SCD, in order to reduce the complications and disability associated with this disease. Additionally, the attention of physicians treating patients with SCD must be drawn to the possibility of AVN in order to prevent or avoid this serious complication, especially in male patients who are presented with frequent pain crises. By knowing the risk factors, patients with symptomatic SCD who are at risk for AVN can be identified and additional evaluations can be performed [24].

\section{Conclusion}

No significant relationship was found between AVN and age in both SCTh and SCA, also no significance was found between SCA and sex. However, in SCTh a significant relationship was detected between AVN and sex, where males had more tendency to develop AVN. Female patients with SCTh had less tendency to develop AVN than the female patients with SCA. The higher HbF in SCA than in SCTh might be due to hydroxyurea treatment which could decreased the AVN incidence in SCA. The AVN prevalence in this study was more than in other studies that could be because of bone scintigraphy technique used which is the most sensitive technique which could detect the silent and the multifocal AVN in SCD.

\section{Disclosure}

No conflicts of interest

\section{Acknowledgment}

We are grateful to the staff of the nuclear medicine department in Amiri Hospital and Mubarak Al-Kabeer Hospital for their efforts in performing scintigraphy. 


\section{References}

1. Ilesanmi OO (2013) Gender differences in sickle cell crises: Implications for genetic counseling and psychotherapy. J Psychol Psychother 3: 10.4172/21610487.1000123.

2. Adewoyin AS (2015) Management of sickle cell disease: A review for physician education in Nigeria (Sub-Saharan Africa). Anemia 2015: 10.1155/2015/791498.

3. Milner PF, Kraus AP, Sebes JI, Sleeper LA, Dukes KA, et al. (1991) Sickle cell disease as a cause of osteonecrosis of the femoral head. N Engl J Med 325: 1476-81.

4. Lavernia CJ, Sierra RJ (2000) Core decompression in atraumatic osteonecrosis of the hip. J Arthroplasty 15: 171-8.

5. Prasad R, Hasan S, Castro O, Perlin E, Kim K (2003) Long-term outcomes in patients with sickle cell disease and frequent vaso-occlusive crises. Am J Med Sci 325: 107-9.

6. Osunkwo I, Hodgman EI, Cherry K, Dampier C, Eckman J, et al. (2011) Vitamin D deficiency and chronic pain in sickle cell disease. Br J Haematol 153: 538-40.

7. Clarke HJ, Jinnah RH, Brooker AF, Michaelson JD (1989) Total replacement of the hip for avascular necrosis in sickle cell disease. J Bone Joint Surg Br 71: 465-70.

8. Ballas SK (2001) Iron overload is a determinant of morbidity and mortality in adult patients with sickle cell disease. Semin Hematol 38: 30-6.

9. Akinyoola AL, Adediran IA, Asaleye CM, Bolarinwa AR (2009) Risk factors for osteonecrosis of the femoral head in patients with sickle cell disease. Int Orthop 33: 923-6.

10. Kamble M, Chatruvedi P (2000) Epidemiology of sickle cell disease in a rural hospital of central India. Indian Pediatr 37: 391-6.

11. Legato MJ (2010) Gender-specific aspects of pediatric hematology and oncology. In: Principles of gender-specific medicine (2 $2^{\text {nd }}$ Edn) Elsevier: Academic Press, USA.

12. Ashley-Koch A, Murphy CC, Khoury MJ, Boyle CA (2001) Contribution of sickle cell disease to the occurrence of developmental disabilities: a populationbased study. Genet Med 3: 181-6.

13. Almeida A, Roberts I (2005) Bone involvement in sickle cell disease. Br J Haematol 129: 482-90.

14. Oniyangi O, Omari AA (2006) Malaria chemoprophylaxis in sickle cell disease. Cochrane Database Syst Rev 18: CD003489.

15. Lafforgue P (2006) Pathophysiology and natural history of avascular necrosis of bone. Joint Bone Spine 73: 500-7.

16. Lee RE, Golding JS, Serjeant GR (1981) The radiological features of avascular necrosis of the femoral head in homozygous sickle cell disease. Clin Radiol 32: 205-14.

17. Ware HE, Brooks AP, Toye R, Berney SI (1991) Sickle cell disease and silent avascular necrosis of the hip. J Bone Joint Surg Br 73: 947-9.

18. Koduri PR, Agbemadzo B, Nathan S (2001) Hemoglobin S-C disease revisited clinical study of 106 adults. Am J Hematology 68: 298-300.

19. Currò G, Meo A, Ippolito D, Pusiol A, Cucinotta E (2007) Asymptomatic cholelithiasis in children with sickle cell disease: early or delayed cholecystectomy? Ann Surg 245: 126-9.

20. Powars DR, Hiti A, Ramicone E, Johnson C, Chan L (2002) Outcome in hemoglobin SC disease: a four-decade observational study of clinical, hematologic, and genetic factors. Am J Hematol 70: 206-15.

21. Al-Jafar H, AlFeeli M, Alhajri FA, Abdullah TA, Al-Shemmeri E (2016) Bone Scintigraphy Scanning Safety and Necessity for Silent and Multifocal Osteonecrosis in Sickle Cell Disease. J Hematol Thromb 2: 1-4.

22. Adekile AD (2011) Limitations of $\mathrm{Hb} F$ as a phenotypic modifier in sickle cell disease: study of Kuwaiti Arab patients. Hemoglobin 35: 607-17.

23. Ballas SK, Kesen MR, Goldberg MF, Lutty GA, Dampier C, et al. (2012) Beyond the definitions of the phenotypic complications of sickle cell disease: an update on management. ScientificWorldJournal 2012 10.1100/2012/949535.

24. Mukisi-Mukaza M, Saint Martin C, Etienne-Julan M, Donkerwolcke M, Burn ME, et al. (2011) Risk factors and impact of orthopaedic monitoring on the outcome of avascular necrosis of the femoral head in adults with sickle cell disease: 215 patient's case study with control group. Orthop Traumatol Surg Res 97: 814-20.

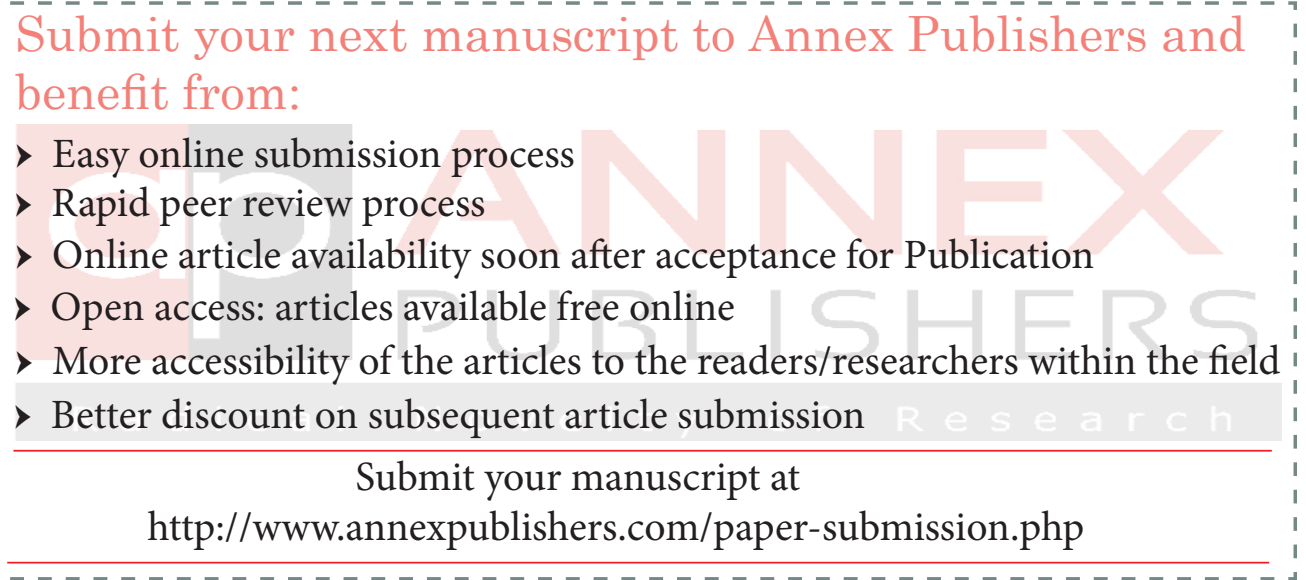

